M. Abedrabbo, M. Wang, P. Tielens, F. Z. Dejene, W. Leterme, J. Beerten and D. Van Hertem, "Impact of DC grid contingencies on AC system stability," 13th IET International Conference on AC and DC Power Transmission (ACDC 2017), Manchester, UK, Feb. 14-16, 2017, 7 pages.

Digital Object Identifier: http://dx.doi.org/10.1049/cp.2017.0025

URL (IET Digital Library):

http://digital-library.theiet.org/content/conferences/10.1049/cp.2017.0025

URL (IEEE Xplore Digital Library):

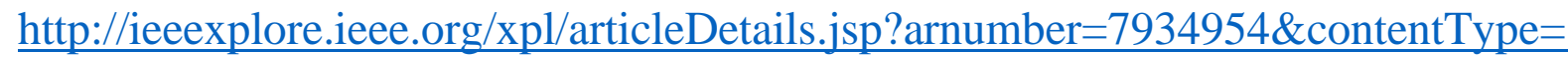
Conference+Publications

(C) 2017 IET. This paper is a postprint of a paper submitted to and accepted for publication in Proc. IET International Conference on AC and DC Power Transmission 2017 and is subject to Institution of Engineering and Technology Copyright. The copy of record is available at IET Digital Library. 


\title{
Impact of DC grid contingencies on AC system stability
}

\author{
Abedrabbo, M. $\ddagger$, Wang, M. $\ddagger$, Tielens, P. $\ddagger$ Dejene, F.Z. $\ddagger$, Leterme, W., Beerten, J. and Van Hertem, D. \\ ELECTA, Department of Electrical Engineering (ESAT), University of Leuven (KU Leuven), Leuven, Belgium and \\ EnergyVille, Genk, Belgium \\ $\ddagger$ Joint first authors
}

Keywords: AC/DC grids, AC system stability, DC contingencies, DC grid protection strategy.

\begin{abstract}
In order to reliably and safely operate future multi-terminal HVDC grids, different protection strategies are currently being developed and presented in the literature. Especially for large DC grids, it is important to investigate how the choice of the protection strategy influences AC system stability during DC fault clearance and system restoration. This paper contributes to the understanding of these issues by giving an overview of the different protection strategies and their influence on frequency and transient stability. DC grid protection requirements are defined, based on the AC system constraints related to the level and duration of power transfer loss.
\end{abstract}

\section{Introduction}

High voltage direct current (HVDC) technology is expected to play a crucial role in the development of future power systems. Especially for transporting large amounts of power over long distances, for connecting different asynchronous zones or to connect offshore wind farms with mainland systems, HVDC is the preferred option as it offers some benefits, e.g. the simpler use of long cables and its controllability compared to AC technology [1].

To this day, HVDC is mostly being used for point-to-point connections. However, by interconnecting several HVDC links, a multi-terminal DC grid is formed which can be embedded into a single $\mathrm{AC}$ grid or linking multiple asynchronous AC systems. Voltage source converter (VSC) HVDC is considered to be the most suitable converter technology for building such a grid since it allows the connection of weak islanded grids and makes it feasible to easily build and control parallel extensions.

The gradual expansion of the existing point-to-point VSC HVDC links toward DC grids poses new challenges that need to be studied. In this paper, the impact of DC grid contingencies on the stability of the associated AC systems is investigated. The level of impact is influenced by the size and structure of the DC grid, the structure of the associated AC grids, the type and duration of the DC Grid contingencies, as well as the type of DC grid protection strategy chosen.
Depending upon the required fault clearing time, the applied converter technology (fault blocking or non-fault blocking) and the available DC grid protection equipment, multiple protection strategies can be adopted to detect and interrupt DC faults, disconnect the affected elements or protection zone and to ensure a stable operation of the remaining system.

From an AC system's perspective, the contingency and accompanied protection strategy could lead to a temporary or permanent power imbalance as well as loss of AC voltage support to the associated AC grids. Each of these elements can impact the AC system stability.

The aim of this work is therefore to shed light on the impact of HVDC grid contingencies on the AC system stability, as well as how this relates to the choice of the DC grid protection strategies.

The structure of the remainder of the paper is as follows: It starts by giving an overview of the different DC contingencies and the AC system stability in section 2. The impact of loss of power transfer from the DC grid on the AC grid frequency stability is briefly demonstrated. Thereafter, in sections 3 and 4 , the different strategies to clear a fault in the DC grid and to restore the system after an outage are addressed and it is described how the power exchange with the $\mathrm{AC}$ system is affected for each protection strategy chosen, in terms of loss of maximum power transfer and the duration. Finally, conclusions and recommendations for future research are given in section 6 .

\section{DC grid contingencies and AC system stability}

\subsection{DC contingencies}

A DC contingency can be defined as a non-functionality of a component within the DC grid such as converter, DC transmission line or busbar, or a non-functionality of the DC grid itself. In this study, the impact of DC contingencies such as converter outages and DC transmission line faults on AC system stability are analyzed. The impact of DC contingencies on AC system stability depends on the topology of both the AC and DC system, the contingency type and the employed AC and DC protections.

Future DC grids are expected to be built step by step from ex- 
isting point-to-point links to form small separate DC grids, and grow into large-scale supergrids through interconnections [1]. Thus the connection between AC and DC systems are assumed to have various forms, such as DC grids embedded within one synchronous AC grid or alternatively DC grids interconnecting different synchronous AC grids, including offshore wind farms. Fig. 1 gives an example of such a possible connection between the AC and DC grid, where a DC grid is connected with three $\mathrm{AC}$ systems. $A C_{2}$ and $A C_{3}$ are asynchronous systems, while $A C_{1}$ has three synchronous areas. We will use Fig. 1 to explain the problems encountered in the $\mathrm{AC}$ grids during DC contingencies.

- Outage of a converter: The power exchange of the converter at the associated node is lost in case of a converter outage. This power exchange is compensated through power droop controls of the other converters in the DC grid, resulting in a near equal change in those converters. If all converters are connected to one synchronous zone, this can be considered as loss of power transfer. If multiple zones are involved, a positive or negative power imbalance will also be created depending on the direction of the exchange.

- DC faults: The impact of a DC fault on the AC and DC grids is directly related to the adopted DC protection strategy. For an AC grid with an embedded DC grid, a DC fault will lead to a temporary loss of power transfer regardless of the adopted DC protection strategy. However, the severity (amount and duration) will depend on the relative size of the DC grid and the DC protection strategy. In the case of a dc grid connecting asynchronous AC grids, a DC fault can result in loss of power in-feed/load, loss of power transfer or combination of both depending on the fault location and adopted DC protection strategy.

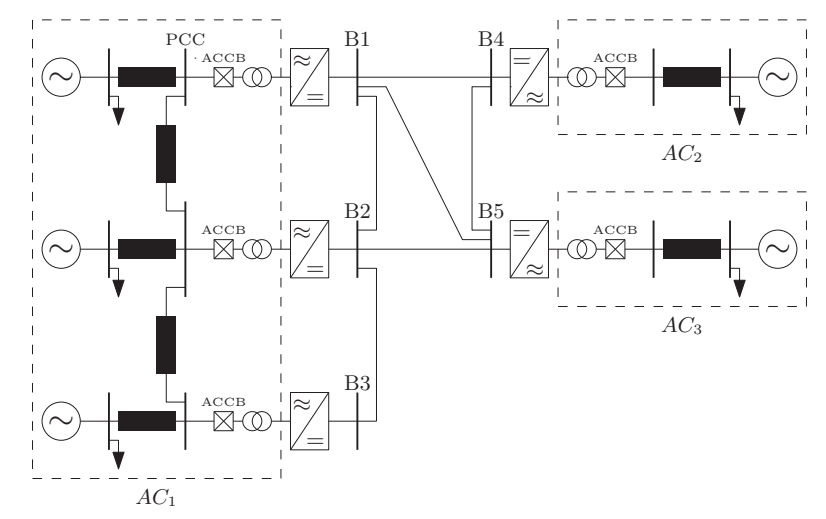

Fig. 1: Example of AC and DC system configuration.

\subsection{Impact on AC system stability}

The stability of an AC power system is in general defined as the ability of the system to regain a state of operating equilibrium after being subjected to a disturbance, with most system variables bounded so that the entire system remains intact [2]. Looking at the system variable in which the instability can be observed, power system stability is further classified into voltage, frequency and rotor angles stability. In this work, the main focus is on these latter two forms which are linked to the electromechanical dynamics within a power system due to the oscillation of the rotating masses of the generators following a disturbance. These dynamics are mainly influenced by the active power transfers in the system and can, due to the inertia provided by the synchronous machines, be considered relatively slow in nature compared to the dynamics within the DC grid. The time frame of interest is mostly in the order of hundreds of milliseconds to seconds.

For point-to-point DC cable links between two distinct AC systems, a contingency at the DC side will always result into a permanent loss of power transfer to the AC side. Considering DC grids on the other hand, the faulty DC component can be isolated and the total power exchange with the AC system may be rapidly restored depending on the applied protection, control strategy and the capacity of the remaining grid.

These fast changes in the power transfer at different terminals might however entice badly damped modes which lead to large rotor angle oscillations between groups of generators that are electrically located far apart from each other within one synchronous zone, possibly resulting in a loss of synchronism. Different factors such as the network configuration, the type and control of the generators, their initial loading and the control of the converters will have an influence.

Considering frequency stability, the network configuration is of less importance and the frequency response for a certain power loss in a synchronous zone due to a DC contingency is determined by the inertia and governor control of the operating power plants, the type of load as well as the behavior of other power electronic coupled generation units. For pointto-point connections, a limit is often imposed on the maximum power injection in order to keep the frequency within predefined boundaries in case the power transfer is lost. For a DC grid with multiple terminals connected to the same synchronous zone, this limit could be increased as the power imbalance within the system can be (partially) restored by altering the power setpoints of the remaining converters.

These phenomena are illustrated in Fig. 2. For the results in Fig. 2, a simple power system equivalent for frequency control analysis is used in order to model the frequency response following a sudden loss of active power infeed $\left(\triangle P_{H V D C}\right)$ from the DC grid during a certain amount of time, represented by $\left(\Delta T_{H V D C}\right)$ as shown in Figure 2(a) [3]. The minimum frequency and rate of change of frequency (ROCOF) directly after the event are given in Figure 2 for different inertia values $(H)$. The total equivalent droop $(R)$ and primary control time constant is taken equal to respectively 0.05 and 1s. Furthermore, it is assumed that sufficient primary reserve is available to cope with the simulated loss of power from the DC system.

As expected, the ROCOF predominantly depends on the power 
imbalance and system inertia. By contrast, the minimum frequency can be increased by rapidly restoring the power exchange with the system. An important consideration is that, to increase the frequency compared to the case of permanent power loss, the restoration of power should occur within a certain time after the contingency, as indicated by the dashed line in Figs. 2(c) and 2(d). Especially for systems with low inertia, this time period is very short, possibly imposing a limit to the maximum available time to restore the DC system.

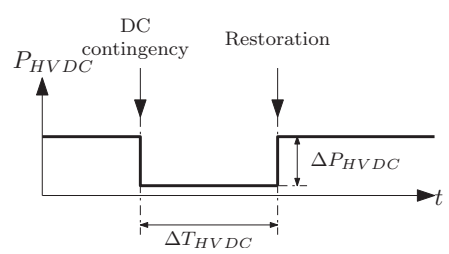

(a) Total active power imbalance profile applied to the AC system

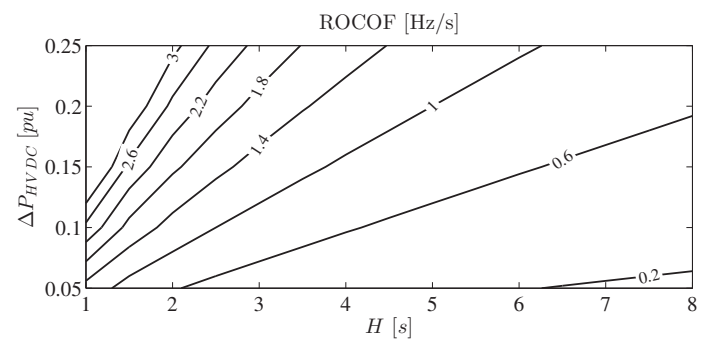

(b)

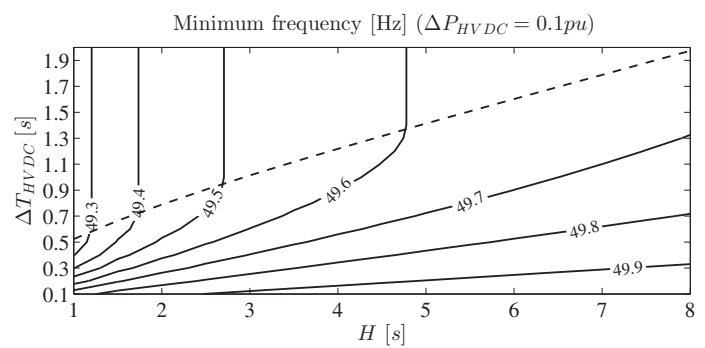

(c)

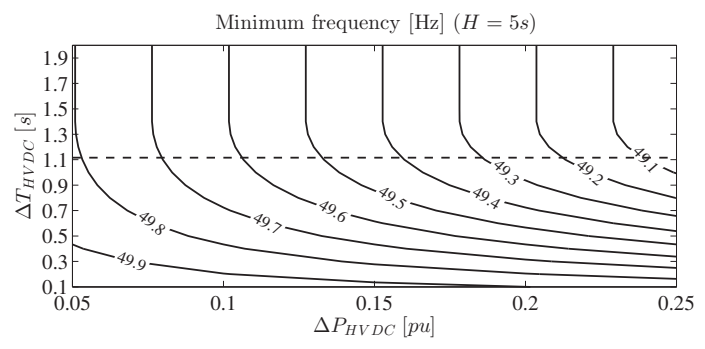

(d)

Fig. 2: Minimum and rate of change of frequency (ROCOF) as function of system inertia, magnitude and duration of power imbalance (system droop $R=0.05$ and equivalent primary control time constant $T=1 \mathrm{~s}$ ), system base is taken equal to the total generation capacity.

\section{DC network fault clearance and restoration}

In the literature, several DC fault clearing strategies are proposed and can be classified as selective, partly-selective and non-selective strategies [4]. In this paper, we focus on the selective and non-selective strategies, since the impact of partlyselective strategies, such as grid-splitting, can be considered as intermediate.

\subsection{Selective protection strategy using DC circuit breakers}

The selective DC fault protection strategy is similar to what is currently been done in $\mathrm{AC}$ transmission system, i.e., isolating only the faulted line by utilizing DC circuit breakers. The total time available for fault clearing typically is in the order of several milliseconds due to the high rate of rise and large steady-state fault current [5]. In the literature, both unit [6,7] and non-unit protection schemes [8-11] for meshed HVDC grids have been proposed. Because of the requirement on the speed of operation, non-unit schemes and unit schemes with fast communication are considered to be applicable in HVDC grids protection.

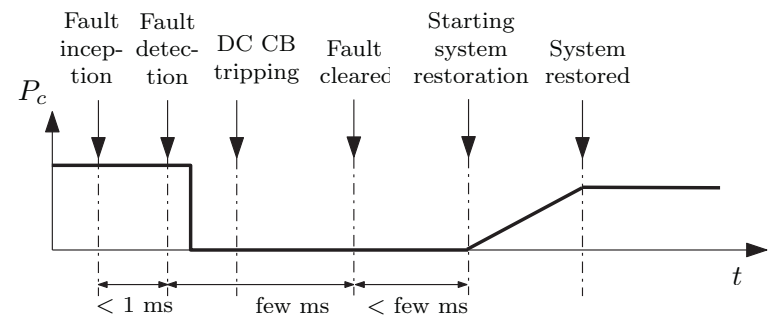

Fig. 3: Selective protection strategy using fast DC circuit breakers.

Fig. 3 shows the steps and time scales involved in a selective protection strategy considering non-unit fault detection algorithms [8], [10], and [11]. Upon arrival of the incident wave at the relay position, it takes less than 1 millisecond to detect and discriminate the faulted line by currently proposed algorithms. Then the tripping signal will be sent to the designated DC circuit breaker. The operating time of hybrid DC circuit breakers is in the range of $2 \sim 5 \mathrm{~ms}$ [12], [13], and $8 \sim 10 \mathrm{~ms}$ for active resonant DC circuit breakers [14]. Once the fault is cleared by the circuit breakers, the DC grid is ready to be restored.

Depending on the passive elements (e.g. fault current limiting inductor), the fault location, and the operating speed of the DC circuit breaker, the converters close to the fault location may be temporarily blocked during the fault clearing process. If the converters, especially the ones at the healthy terminals are blocked, algorithms or sequences in order to de-block and resynchronize the converters are necessary in order to restore the DC grid. The duration for power restoration is typically in the order of milliseconds without converter blocking, and may be longer if converters are blocked during the fault clearing process. The impact on the AC system stability, therefore, is 
expected to be insignificant if fast power restoration can be achieved.

\subsection{Non-selective protection strategy using AC circuit breakers for fault clearing}

In a second strategy, the fault current is interrupted by opening all circuit breakers installed at the AC side of the converters. A communication-less protection scheme as for instance presented in [15] can be adopted in order to reliably clear the fault, isolate the faulted DC line and subsequently restore the entire system.

In this case, only relatively cheap DC switches are installed at both ends of each DC line and no DC circuit breakers are required which reduces the investment cost of building multiterminal HVDC grids [16]. However, this strategy leads to long fault clearing times as it may take up to a few cycles of the fundamental $\mathrm{AC}$ frequency to interrupt the fault current by the AC circuit breaker (40-80 ms) [4]. Additionally, due to the non-selective fault interruption method, the entire DC network will be de-energized before the fault is cleared [17].

In Figure 4, the different steps and time scales involved in this method are schematically presented. Also the variation of the active power of a single converter $\left(P_{c}\right)$ and the AC voltage $\left(U_{P C C}\right)$ at the point of common coupling (see also Figure 1$)$ is roughly illustrated.

When the fault occurs, the current in each converter will not vary instantaneously, but a very small time delay exists before the fault reaches each converter due to the finite speed of the traveling wave from the fault location to the converter terminals. This time delay depends mainly on the fault location, grid layout and network parameters and is mostly in the order of milliseconds. Once the converter detects the fault and the current reaches a certain threshold value, the converter protection will block the converter in order to prevent damage the IGBTs, which have only a limited overcurrent capability. At the same time, a tripping signal is send to the ACCBs.

Due to the blocking of the IGBTs, the current is commutated to the anti-parallel diodes and the converter becomes an uncontrolled rectifier (assuming a converter with half-bridge technology). This leads to a large infeed from the AC system which will, together with the current from the discharge of the DC capacitors and cables, determine the total fault current. Eventually, the fault current reaches a steady state value, predominantly determined by the infeed from the AC system [17]. The AC terminal voltage has by now dropped significantly, to a value approximately equal to the voltage drop across the phase reactor and arm inductor.

Within the time period between the fault detection and operation of the AC circuit breaker, the faulted DC branch is identified using local current and voltage measurements [15]. As soon as the current through the faulted line has dropped to zero, the fault is isolated by opening the designated DC switches. In the meantime, also the voltage at the $\mathrm{AC}$ side is raised or

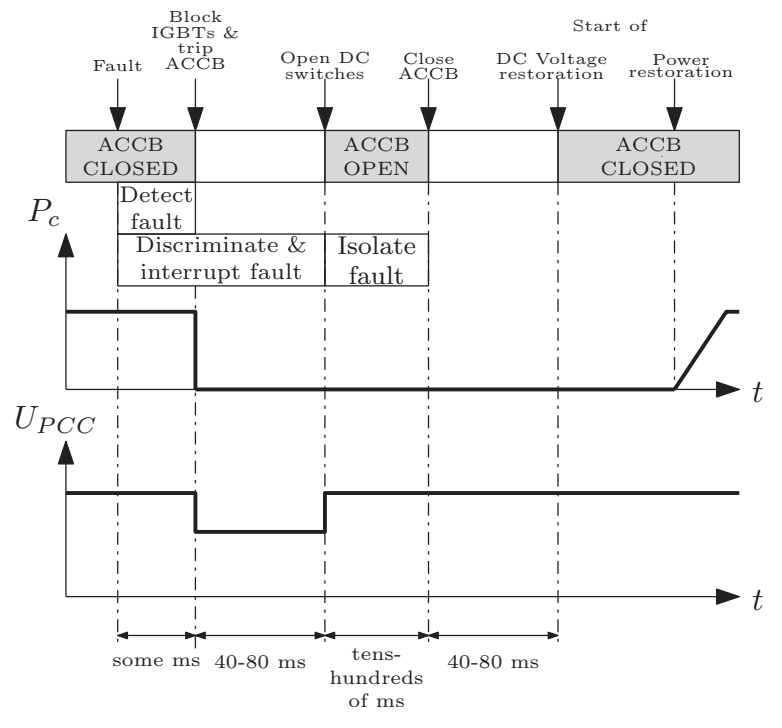

Fig. 4: ACCB strategy.

restored to its nominal value, depending on the voltage regulation capability of the AC system. Finally, the DC grid is reconnected by the ACCBs in order to regain pre-fault system operation. The time required to restore the system is highly depending on the applied converter control and system layout. However, as it corresponds to a complete DC grid restart including ramping up the power, it can possibly take seconds or even minutes to fully restore the system.

From the point-of-view of an AC system, the DC grid fault can be considered equivalent to multiple short-circuits at each converter terminal as soon as the IGBTs of the converters are blocked [16]. Due the voltage drop within the AC system and the long fault clearing times, transient stability issues can therefore occur, possibly resulting in the loss of synchronism of the synchronous generators connected to the system.

Furthermore, since the power exchange with the AC system is lost during several seconds, the frequency stability may be jeopardized depending on the strength of the AC system in terms of inertia and on the primary control capability. Large frequency deviations may occur which might result in load shedding or even lead to a complete black-out.

\subsection{Non-selective protection strategy using converters with DC fault blocking capability for fault clearing}

The utilization of the modular multi-level converter (MMC) with DC fault blocking capability in the multi-terminal DC grid represents the third fault clearing strategy considered in this paper. Various converter typologies with DC fault blocking capability were presented in [18] and [19]. The blocking capability of the converter is exploited to control the DC current by inserting negative voltages or to interrupt it by blocking all converters in the grid [20]. As the required time to insert negative voltages by the submodules (to control the DC current) or to turn off the submodules (to interrupt the DC fault 


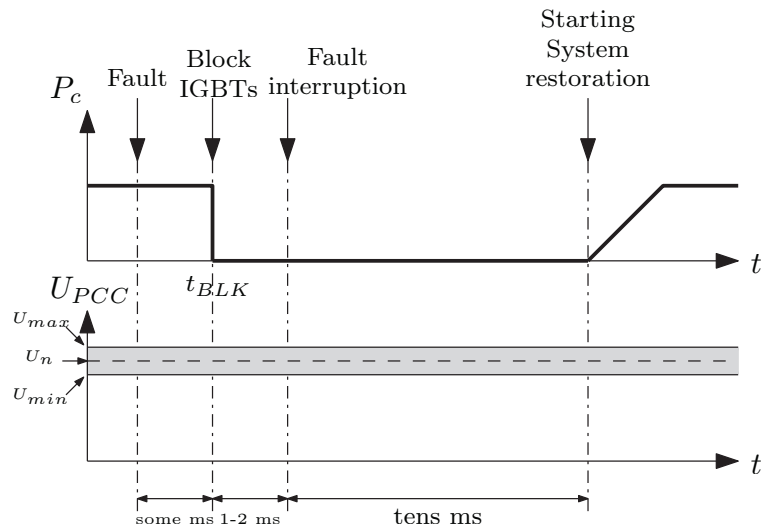

Fig. 5: Fault clearance strategy by converters with DC fault blocking capability.

current) is within 1-2 ms, the fault current interruption is in the range of 1-2 ms as well [21]. Hence, fast DC circuit breakers are not required. However, residual current breakers (RCB) can be included at both ends of each DC line, to clear the residual currents after the converters' blocking and to isolate the faulty line in order to restore the system safely [22]. Similar to the AC circuit breakers strategy, the protection system is used for this strategy to detect and identify the faulty line.

Figure 5 demonstrates the stages of fault clearance by converter blocking. Furthermore, it shows two sketches for the exchanged active power $\left(P_{c}\right)$ and the $\mathrm{AC}$ voltage at the point of common coupling $\left(U_{P C C}\right)$. The fault clearance period is divided into three intervals; the first interval represents the time before the detection of the fault, while the second interval starts at the instant of detection and blocking of the converter. The restoration of the system operation is initiated in the third interval.

During the first interval, the voltage at the point of common coupling and the exchanged active power will not be influenced, even after the arrival of the fault wave at the DC terminal of the converter, since the converter is still in operation [21]. However, the submodules will be turned off once the current in the converter exceeds a specific threshold at $t_{B L K}$, and the converter turns to blocking mode. Consequently, the power drops to zero as illustrated in Figure 5. Thus the AC system will see the converter blocking as a disconnection of a heavy load or as a disconnection of a generation unit.

Although the shut down of the entire DC grid is similar to the previous strategy, the time scale of current interruption and restoration is much shorter compared with the AC circuit breaker strategy. The starting instant of system restoration depends on the size of the HVDC grid and the speed of the RCBs to isolate the faulty part. In [22], two types of RCBs were examined (RCB with $2 \mathrm{~ms}$ and $30 \mathrm{~ms}$ opening time). The required time to unblock the converters and restore the grid operation is directly proportional to the the speed of the installed RCBs.

The capability of this type of converters is not limited to inter- rupt the DC current, it can be extended to supply the required reactive power to the $\mathrm{AC}$ grid (STATCOM operation mode) even during DC side faults by reducing the exchanged power to zero and keeping the converter in operation as proposed in [19] and [23] to support the AC grid.

\section{Converter outage and droop control}

Contrary to a fault on the DC grid which can be cleared using one of the aforementioned strategies, a fault within the converter or on its DC bus bar will mostly result in a permanent loss of the converter. This loss is immediately reflected by an increase or decrease in the DC voltage due to the (dis)charging of the cables and DC capacitors.

In order to stabilize this DC voltage within the system, the power imbalance may be compensated by a single (slack) converter or by multiple converters using a distributed DC voltage droop control scheme [24]. Different converters will in this case jointly react upon the changing DC voltage by altering their power set points, similar to the primary control action used in AC systems.

The time scale though of the DC voltage stabilization is in the range of tens of millisecond, i.e. a couple of orders of magnitude smaller than the primary control action within the AC system. The power sharing amongst the converters participating in this droop control can further be tuned by changing the droop constants.

In case each converter is linked to a separate AC system, the outage of a converter and the accompanied control action of the remaining converters will therefore lead to a fast variation in the power output. Hence, from the point-of-view of each AC system, it can be considered equivalent to the loss of a single load or generation unit which will directly affect the AC system frequency. Weak AC systems with low inertia, insufficient reserve capacity or slow governor control will encounter large frequency transients, leading to load shedding or disconnection of renewable generation units, possibly aggravating the initial frequency event. Therefore, in order to ensure the frequency to stay within the boundaries after an outage, a limit may be imposed to the maximum power that is transferred in steadystate.

When considering an HVDC grid in which multiple DC converters are linked to one interconnected $\mathrm{AC}$ system, the total power exchange with the DC grid may be rapidly restored, improving the frequency response after the outage as shown in section 2.2. However, due to the parallel paths that exist at the $\mathrm{AC}$ and DC side, complex interactions will occur between both systems. Therefore, care should been taken in selecting the droop constants in order to not compromise system stability. To this end, a methodology which optimizes the droop settings in order to minimize the adverse effects on the AC system as for instance presented in [25] can be applied. 


\section{Discussion}

As described in the previous sections of the paper, the time frame of DC contingencies is much shorter than those in AC systems. Furthermore, as illustrated in section 2.2, the minimum frequency during a contingency can be increased if the loss of power is rapidly restored. However, the current AC grid code is determined based on permanent loss of power infeed which might not be the most appropriate for the future overlay AC/DC grids. One possible suggestion is given in Fig. 6, where the maximum allowed loss of power infeed $P_{\max }$ is in accordance with the current AC grid code. However, this level could be higher if the power could be restored in shorter times. The maximum loss of power infeed could be defined as multiple steps, transient loss (restoration within $t_{1}$ e.g. less than one cycle), temporary loss (restoration within $t_{2}$ e.g. hundreds ms), and permanent loss (restoration longer than $t_{3}$ ). In this paper, an equivalent single machine model is used to illustrate the impact of various durations; however, more detailed models are needed to define the different characteristics shown in Fig. 6 for a given system. Consequently, once the maximum loss of power infeed and duration is defined from the surrounding $\mathrm{AC}$ systems, the protection strategy of a DC grid would have to be designed to fulfill these requirements.

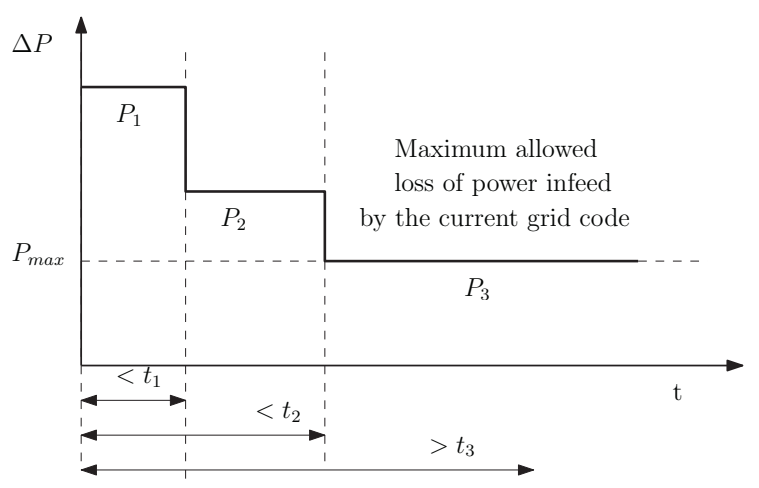

Fig. 6: Possible future AC grid code: loss of power infeed and duration.

\section{Conclusions}

The stability of the AC system during DC contingencies is mainly influenced by the impact of these contingencies in terms of power imbalance and duration. These are directly influenced by the adopted DC protection strategy, the type of contingency and its location. Three fault clearance strategies were discussed in terms of time scale and their impact on the AC grid stability.

The selective protection strategy might not have an impact on the frequency and transient stability, as fast DC circuit breakers are used to isolate the faulty part. The duration of the fault detection, clearance and system restoration is in the range of tens of milliseconds which is considered to be too short to have an influence on the AC grid stability.
On the contrary, the AC circuit breakers strategy for fault clearance results in de-energization of the DC grid for long period of time. Due to the long period of fault clearance and system restoration, the AC grid can encounter transient stability problems if the size of the DC system is relatively large compared to the $\mathrm{AC}$ system.

Considering the converters with DC fault blocking capability, the restoration time of the whole system depends on the size of the DC grid. In addition, the speed of operation of the installed RCBs plays a main role in determining the required time to initiate the system restoration. However, the restoration time of non-selective strategy using converters with DC fault blocking capability is shorter than non-selective strategy using AC circuit breakers.

Moreover, the impact of the converter outage depends on the AC/DC grid topology. In case of each converter connected to a separate AC grid, the maximum amount of power transfer loss by each converter should be limited to ensure the frequency stability during the converter outage, while the droop constants should be carefully selected in case of embedded DC grid within one synchronous $\mathrm{AC}$ grid or multiple converters connected to one AC grid, in order to minimize the impact on the AC system.

Finally, a future AC grid code has been proposed which defines the maximum level and duration of power transfer loss based on the AC system constraints. Further research is however required to determine the specific shape of this curve which will set a limit to the maximum available time to restore the power transfers between the AC and DC grid.

\section{Acknowledgment}

The work of Mudar Abedrabbo is funded by Mitsubishi Electric Corporation. The work of Firew Dejene, Willem Leterme and Mian Wang is funded by Horizon 2020 PROMOTioN (Progress on Meshed HVDC Offshore Transmission Networks) under grant agreement No 691714. The work of Jef Beerten is funded by a research grant of the Research Foundation-Flanders (FWO).

\section{References}

[1] D. Van Hertem and M. Ghandhari, "Multi-terminal VSC HVDC for the European supergrid: Obstacles," Renewable and Sustainable Energy Reviews, vol. 14, no. 9, pp. 3156-3163, 2010.

[2] P. Kundur, J. Paserba, V. Ajjarapu, G. Andersson, A. Bose, C. Canizares, N. Hatziargyriou, D. Hill, A. Stankovic, C. Taylor, T. V. Cutsem, and V. Vittal, "Definition and classification of power system stability ieee/cigre joint task force on stability terms and definitions," IEEE Transactions on Power Systems, vol. 19, pp. 1387-1401, Aug 2004. 
[3] G. C. Tarnowski, Coordinated Frequency Control of Wind Turbines in Power Systems with High Wind Power Penetration. PhD thesis, Technical University of Denmark, 2011.

[4] W. Leterme and D. Van Hertem, "Classification of fault clearing strategies for hvdc grids," in Cigre Conference Lund, 2015.

[5] D. Van Hertem, M. Ghandhari, J. B. Curis, O. Despouys, and M. Andrée, "Protection requirements for a multi-terminal meshed DC grid," in Cigrè International Symposium THE ELECTRIC POWER SYSTEM OF THE FUTURE Integrating supergrids and microgrids, Cigré, sep 2011.

[6] J. Descloux, Protection contre les courts-circuits des réseaux à courant continu de forte puissance. $\mathrm{PhD}$ thesis, Université de Grenoble, Grenoble, France, Sept. 2013.

[7] N. Johannesson and S. Norrga, "Longitudinal differential protection based on the Universal Line Model," in Industrial Electronics Society, IECON 2015 - 41st Annual Conference of the IEEE, (Yokohama, Japan), pp. 001091-001096, 9-12 Nov. 2015.

[8] K. De Kerf, K. Srivastava, M. Reza, D. Bekaert, S. Cole, D. Van Hertem, and R. Belmans, "Wavelet-based protection strategy for DC faults in multi-terminal VSC HVDC systems," IET Gener. Transm. Distrib., vol. 5, pp. 496-503, Apr. 2011.

[9] N. Johannesson, S. Norrga, and C. Wikström, "Selective Wave-Front Based Protection Algorithm for MTDC Systems," in IET DPSP 2016, (Edinburgh, UK), p. 6 pages, 7-10 Mar. 2016.

[10] J. Sneath and A. D. Rajapakse, "Fault Detection and Interruption in an Earthed HVDC Grid using ROCOV and Hybrid DC Breakers," vol. 31, pp. 973-981, June 2016.

[11] W. Leterme, J. Beerten, and D. Van Hertem, "Non-unit protection of HVDC grids with inductive dc cable termination," vol. 31, pp. 820-828, Apr. 2016.

[12] J. Häfner and B. Jacobson, "Proactive hybrid hvdc breakers-a key innovation for reliable hvdc grids," in Proc. Cigré Bologna Symp., (Bologna, Italy), p. 8 pages, 13-15 Sep. 2011

[13] C. C. Davidson, R. S. Whitehouse, C. D. Barker, J.-P. Dupraz, and W. Grieshaber, "A new ultra-fast HVDC Circuit breaker for meshed DC networks," in Proc. IET $A C D C$, (Birmingham, UK), p. 7 pages, 10-12 Feb. 2015.

[14] K. Tahata, S. El Oukaili, K. Kamei, D. Yoshida, Y. Kono, R. Yamamoto, and H. Ito, "HVDC circuit breakers for HVDC grid applications," p. 9 pages, 2729 May 2015.
[15] L. Tang and B. T. Ooi, "Locating and isolating dc faults in multi-terminal dc systems," IEEE Transactions on Power Delivery, vol. 22, pp. 1877-1884, July 2007.

[16] G. Li, J. Liang, C. E. Ugalde-Loo, P. Coventry, and J. Rimez, "Dynamic interactions of dc and ac grids subject to dc faults," in 2016 IEEE 8th International Power Electronics and Motion Control Conference (IPEMCECCE Asia), pp. 2627-2633, May 2016.

[17] M. K. Bucher and C. M. Franck, "Contribution of fault current sources in multiterminal hvdc cable networks," IEEE Transactions on Power Delivery, vol. 28, pp. 1796-1803, July 2013.

[18] A. Nami, J. Liang, F. Dijkhuizen, and P. Lundberg, "Analysis of modular multilevel converters with dc short circuit fault blocking capability in bipolar hvdc transmission systems," in Power Electronics and Applications (EPE'15 ECCE-Europe), 2015 17th European Conference on, pp. 1-10, Sept 2015.

[19] M. M. C. Merlin, T. C. Green, P. D. Mitcheson, D. R. Trainer, D. R. Critchley, and R. W. Crookes, "A new hybrid multi-level voltage-source converter with dc fault blocking capability," in AC and DC Power Transmission, 2010. ACDC. 9th IET International Conference on, pp. 1-5, Oct 2010.

[20] E. Spahic, F. Schettler, D. Varma, and J. Dorn, "Impact of the dc technology on transmission grids," in $A C$ and DC Power Transmission, 11th IET International Conference on, pp. 1-7, Feb 2015.

[21] M. Abedrabbo, C. Petino, and A. Schnettler, "Analysis of the behavior of hvdc converter based on fullbridge submodules during dc fault conditions," in 2016 IEEE International Energy Conference (ENERGYCON), pp. 1-6, April 2016.

[22] C. Petino, M. Heidemann, D. Eichhoff, M. Stumpe, E. Spahic, and F. Schettler, "Application of multilevel full bridge converters in hvdc multiterminal systems," IET Power Electronics, vol. 9, no. 2, pp. 297-304, 2016.

[23] C. Karwawita, D. Suriyaarachchi, and M. Mohaddes, "A controlled dc fault clearance mechanism for full-bridge mmc vsc converters," in Cigre Conference Lund, 2015.

[24] J. Beerten and R. Belmans, "Analysis of power sharing and voltage deviations in droop-controlled $\mathrm{dc}$ grids," IEEE Transactions on Power Systems, vol. 28, pp. 4588-4597, Nov 2013.

[25] R. Eriksson, J. Beerten, M. Ghandhari, and R. Belmans, "Optimizing dc voltage droop settings for ac/dc system interactions," IEEE Transactions on Power Delivery, vol. 29, pp. 362-369, Feb 2014. 